\title{
Mesenteric infantile hemangioma: diagnostic work-up
}

\author{
Francesco Esposito ${ }^{1}$, Marco Di Serafino ${ }^{2}$, Rosa Severino ${ }^{3}$, Zukhra Vafina ${ }^{3}$, Dolores Ferrara ${ }^{3}$, Massimo \\ Zeccolini $^{1}$
}

${ }^{1}$ Radiology Department, Santobono Hospital, Napoli, Italy; ${ }^{2}$ Department of Emergency Radiology, San Carlo Hospital, Potenza, Italy; ${ }^{3}$ Radiology Department, "Federico II" University Hospital, Napoli, Italy

Correspondence to: Francesco Esposito. Radiology Department, Santobono Hospital, Mario Fiore st 6, Napoli, Italy. Email: fra.in@libero.it.

\begin{abstract}
A very rare localization of infantile hemangioma (IH) is mesentery. It may manifest with intraluminal or intra-peritoneal bleeding. Here there is the case of a 45-day-old infant, presenting with anemia and poor growth, subsequently referred to an uncommon hemangioma of the mesentery. As well as for most of IH, conservative treatment with only propranolol resulted in the disappearance of the lesion. Here we analyze ultrasound and magnetic resonance (MR) features of this uncommon lesion, trying to underline main characteristics at imaging in order to monitor the response to therapy.
\end{abstract}

Keywords: Ultrasonography; infant; hemangioma; mesentery; propranolol; peritoneal disease

Submitted Jul 11, 2017. Accepted for publication Aug 31, 2017.

doi: 10.21037/qims.2017.09.01

View this article at: http://dx.doi.org/10.21037/qims.2017.09.01

\section{Introduction}

Hemangiomas are benign vascular tumors arising from the capillary endothelium when affected by an increased cell turnover, which can be observed throughout all the human body: skin, muscles, bones and internal organs. They should be distinguished from vascular malformations, which are structural anomalies of capillary, venous, lymphatic and arterial systems that expand in proportion to the child growth and therefore often become clear only after the puberty $(1,2)$. Infantile hemangioma (IH) is the most common vascular tumor of childhood, affecting about $5-10 \%$ of all infants. It usually appears during the first weeks after birth, then there is an intense proliferation and at last gradual spontaneous involution until disappearance in most of cases by $10-12$ years of age $(3,4)$.

\section{Case presentation}

A 45-day-old female newborn was admitted to the Pediatric Hospital affected by inappetence, pale skin and poor growth. Blood sample test showed signs of normochromic normocytic anemia. No fecal occult blood was found.

An abdominal ultrasound examination showed diffused and marked hyperplasia of small bowel mesenteric fat, which also appeared hyperechoic, in particular at the level of central and left portion of the abdomen (Figure 1), with numerous globular-shaped lymph nodes (ranging 5-15 mm in diameter) (Figure 2). Overall, mesenteric fat tissue preponderance over lymph nodes was noticed. Furthermore, color-Doppler study revealed an increased vascularization of the pathologic area (Figure 3) with dislocated and enlarged central abdominal vessels (veins in particular), without signs of thrombosis (Figure 4). Diameter of superior mesenteric artery, superior mesenteric vein and portal vein were 4 , 6.6, and $6.7 \mathrm{~mm}$, respectively. No pathologic thickness of intestinal wall was found.

Contrast-enhanced magnetic resonance imaging (MRI) of the abdomen showed large area of mesenteric fat replaced by a "pseudo-nodular" tissue with some bowel loops enclosed within, which appeared iso-intense on $\mathrm{T} 1$ weighted images and hyper-intense on T2 weighted images. After contrast administration, the pathologic tissue showed strong but slightly inhomogeneous enhancement. Furthermore, bowel resulted displaced as well as the mesenteric vascular axis. An anticlockwise course of an afferent jejunal vein of the superior mesenteric vein was also noticed. There were no signs of ascites neither other 


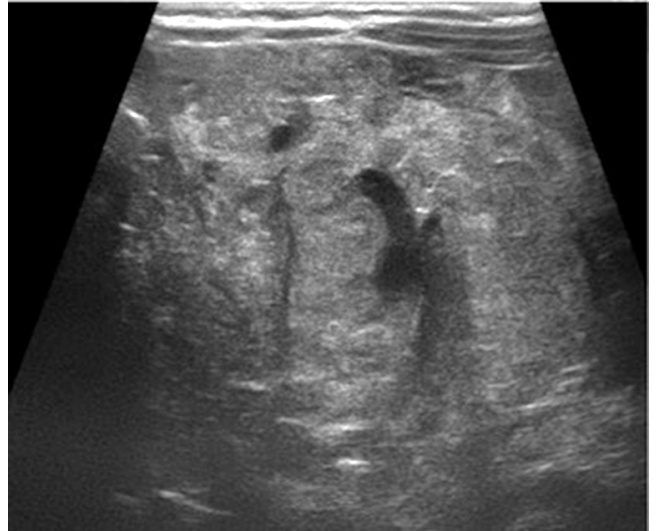

Figure 1 Abdominal ultrasound examination shows diffused and significant thickening of omental fat tissue of small bowel.

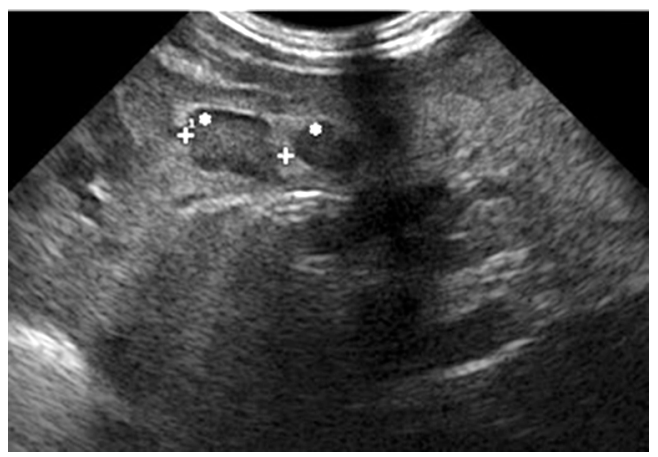

Figure 2 Abdominal ultrasound examination with convex probe shows hyperechoic omental fat and enlarged lymph-nodes (maximum transverse diameter about $15 \mathrm{~mm}$ ) within it (asterisks), the cross signs mark the diameter of the lymph-nodes.

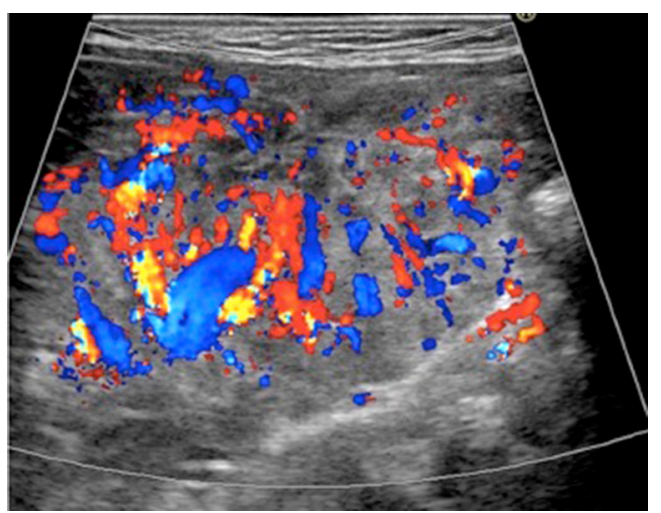

Figure 3 Abdominal color-Doppler ultrasound examination shows high vascularization of thickened, hyperechoic omental tissue.

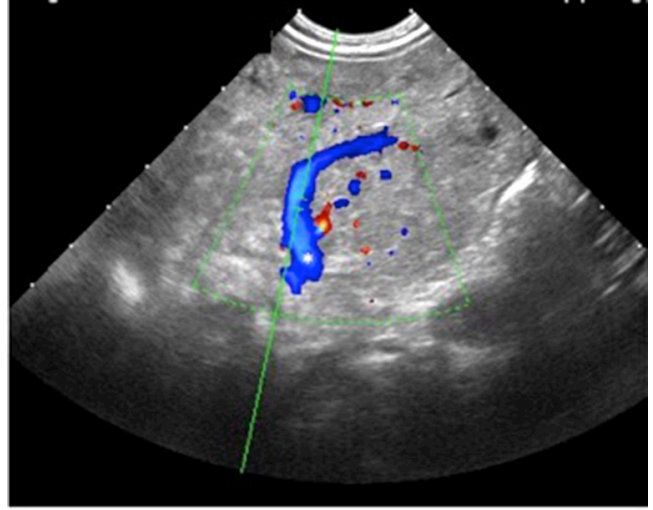

Figure 4 Abdominal color-Doppler ultrasound examination shows enlarged abdominal vessels, veins in particular, which also appeared tortuous (asterisk).

remarkable changes in the abdominal organs (Figure 5).

At last, a diagnostic laparoscopy was performed. This tool was preferred to ultrasound-guided fine needle biopsy because of high risk of hemorrhage from the vessels within the mass. The exploration of the peritoneal cavity revealed a vascular lesion almost completely replacing the mesentery of the small bowel (some jejunal loops and ileum), the sigma and the rectum until the peritoneal edge. In addition, there was marked dilatation of the umbilical vein and of the vessels along the descending colon (Figure 6). Rectocolonoscopy revealed normal mucosa with prominent sub-mucosal vascular reticulum in the recto-sigma area. Intestinal peristalsis appeared preserved. No malignant cells were found at the peritoneal cavity lavage.

A multidisciplinary team meeting concluded for propranolol as the treatment of choice for the patient. At 3 months follow-up of therapy, the young patient showed normal growth without signs of anemia at blood sample. An abdominal ultrasound showed almost restored position of bowel loops, with decreased thickness of the mesenteric pathologic tissue. The diameter of central-abdominal vessels also resulted decreased (superior mesenteric artery: $3.2 \mathrm{~mm}$, superior mesenteric vein: $4 \mathrm{~mm}$, portal vein: $6.4 \mathrm{~mm}$ ). At the same time, a relative increase of lymph nodes diameter was noticed with consequent lower mesenteric tissue/ lymph-nodes volume ratio. A possible comment to this ultrasonographic picture could be "the more and bigger the lymph nodes appear, the less and thinner the mesenteric tissue became". 


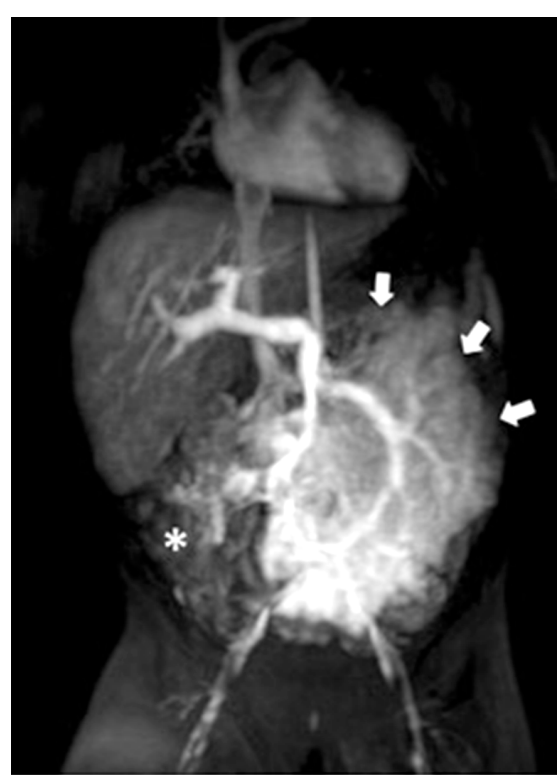

Figure 5 Contrast-enhanced MRI, MIP coronal reconstruction, shows a "pseudo-nodular" tissue with inhomogeneous enhancement (arrows), which replaces omental fat and causes displacement of bowel loops to the peripheral side of the abdomen (asterisk). MRI, magnetic resonance imaging; MIP, maximum intensity projection.

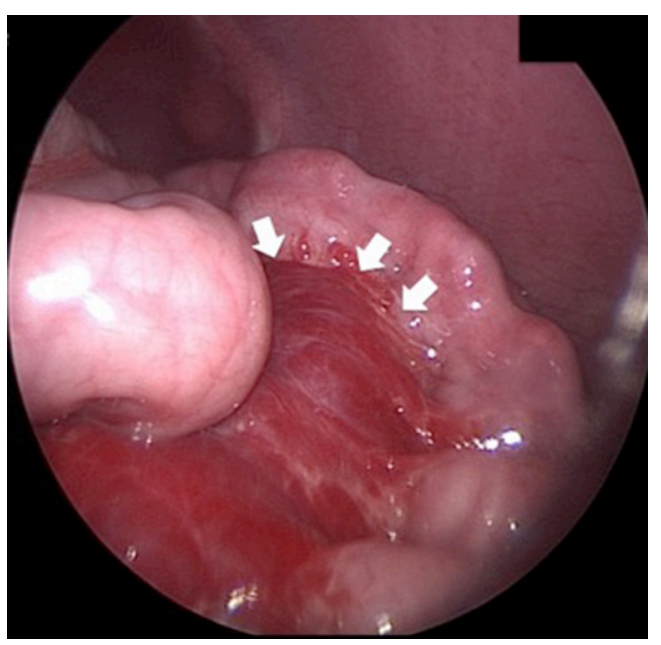

Figure 6 Laparoscopy shows a high vascular lesion replacing almost completely the mesenteric fat of the small bowel (arrows).

\section{Discussion}

Mesentery represents one of the rarest localization of hemangiomas, which may arise either from the bowel wall and the mesentery or the mesentery alone rather than the bowel wall. The clinical presentation is variable, depending on its size, localization and intestinal wall involvement. Growth of mesenteric hemangiomas (MH) into the bowel wall may result in intraluminal bleeding and therefore hematemesis or melena, whereas extra-luminal bleeding may cause hemoperitoneum and therefore result in life-threatening condition. As it happened in our case, when slow, bleeding may manifest with signs and symptoms of anemia (5-8).

Despite several imaging tools may lead to the correct diagnosis, $\mathrm{MH}$ are still often misdiagnosed because of their low incidence in general practice and the difficulties in imaging interpretation.

Usually, ultrasound is the first-line imaging modality in pediatric patients, followed by computed tomography (CT) and/or MRI. Laparoscopy and histology are possibly used when other techniques fail in reaching the diagnosis (5-7).

In our case ultrasound was the first exam, which showed mesenteric pathological changes. Many authors describe the ultrasound appearance of cavernous variant of MH (9-13), which is referred to as "an enormous tumor mass with an inhomogeneous echo pattern" (8) or "solid lobulated mass of variable echogenicity, homogenous or heterogenous" (6). However, according to the new ISSVA classification [2013], this pattern is included among vascular malformations instead of IH (1-4). It could not be found any description of ultrasound findings of mesenteric $\mathrm{IH}$, corresponding to the new ISSVA classification.

In our experience, the ultrasound appearance of $\mathrm{MH}$ does not resemble a tumor mass but rather a pathologic hyperplasic mesenteric tissue spreading within displaced bowel loops. Moreover, displaced and ectasic mesenteric vessels were found. All these features have been subsequently used to evaluate treatment success.

In the diagnostic setting, CT and MRI allow a more precise evaluation of tumor size, its vascularization and possible involvement of adjacent structures. Despite MRI findings may sometimes be ambiguous, because of the presence in the pathologic tissue of changing like fibrosis, edema, hemorrhage, which make difficult the real diagnosis $(9,11)$, in our case MRI findings were quite typical (4) and also helped to evaluate spreading of the pathologic mesenteric tissue and its vascularization.

Generally, the definitive diagnosis of tumors is made up by biopsy and histopathological examination. On the contrary, biopsy of $\mathrm{MH}$ is not strongly recommended because of the high risk of bleeding during the procedure. It is recommended only in particular cases when malignant vascular tumors are suspected. Therefore, a diagnostic 
laparoscopy is usually preferred, as it allows not only to make the diagnosis but also to evaluate the treatment options, which, in the case of $\mathrm{MH}$, include both medical and surgical choice (surgical removal, embolization of the mesenteric vessels, sclerotherapy) (1-4).

It is widely accepted that hemangiomas tend to spontaneous involution, therefore, when very rare malignant variants are excluded (e.g., hemangioendothelioma or angiosarcoma) no treatment is usually required, but only periodical monitoring of the lesion. However, when hemangiomas present with pain or because of their localization may cause visual compromise or airway obstruction, or may induce life-threatening conditions, medical or surgical treatment becomes necessary (1-4).

This was our case, as the young patient showed anemia, inappetence and poor growth. Furthermore, the mesenteric localization was crucial for possible development of lifethreatening intra-abdominal bleeding. The diagnostic laparoscopy excluded the possibility of embolization of the lesion because of its huge size; therefore, a pharmacological treatment was preferred. Among various options (corticosteroids, interferon, b-blockers, cyclophosphamide or vincristine), the treatment with propranolol is considered to be the most effective and relatively safe in case of IH, associated with non-invasive follow-up with ultrasound to confirm lesion regression $(1-4,13,14)$.

After 3 months of therapy, the significant improvement of clinical features, laboratory exams and ultrasound findings led to definitively confirm the diagnosis of $\mathrm{IH}$.

Finally, here we propose some possible ultrasound parameter that could help in providing an objective assessment of treatment success of mesenteric IH. A qualitative analysis includes the evaluation of thickness of the mesenteric pathologic tissue and its relation with adjacent lymph nodes, e.g., in case of treatment success lymph nodes become more evident as the consequence of decrease of hyperplasic mesenteric tissue that "hided" them before; a qualitative evaluation of vascularization of the pathologic mesenteric tissue, with unusual disposition of mesenteric vessels, is also very important. The second parameter is quantitative and includes the evaluation of the size of the abdominal vessels next to the pathologic mesenteric tissue (e.g., superior mesenteric vein, superior mesenteric artery, portal vein). These ultrasound features may allow practitioners to evaluate the effectiveness of therapy of $\mathrm{MH}$ during follow-up.

\section{Acknowledgements}

None.

\section{Footnote}

Conflicts of Interest: The authors have no conflicts of interest to declare.

Informed Consent: Written informed consent was obtained from the patient for publication in this case report and any accompanying images.

\section{References}

1. Bhat V, Salins PC, Bhat V. Imaging spectrum of hemangioma and vascular malformations of the head and neck in children and adolescents. J Clin Imaging Sci 2014;4:31.

2. Nosher JL, Murillo PG, Liszewski M, Gendel V, Gribbin CE. Vascular anomalies: A pictorial review of nomenclature, diagnosis and treatment. World J Radiol 2014;6:677-92.

3. Chen TS, Eichenfield LF, Friedlander SF. Infantile hemangiomas: an update on pathogenesis and therapy. Pediatrics 2013;131:99-108.

4. Lowe LH, Marchant TC, Rivard DC, Scherbel AJ. Vascular malformations: classification and terminology the radiologist needs to know. Semin Roentgenol 2012;47:106-17.

5. Drolet BA, Pope E, Juern AM, Sato T, Howell B, Puttgen KB, Lara-Corrales I, Gilliam A, Mancini A, Powell J, Siegel D, Metry D, Stevenson DA, Grimmer JF, Frieden IJ. Gastrointestinal bleeding in infantile hemangioma: a complication of segmental, rather than multifocal, infantile hemangiomas. J Pediatr 2012;160:1021-6.e3.

6. Massaro M, Suárez T, Huertas C, Cuervo C. Mesenteric hemangiomas. Report of four cases. Rev Colomb Radiol 2012;23:3541-5.

7. Rubinstein JC, Christison-Lagay ER. Successful treatment of visceral infantile hemangioma of the omentum and mesentery with propranolol. J Ped Surg Case Reports 2014;2:302-4.

8. Kazimi M, Ulas M, Ibis C, Unver M, Ozsan N, Yilmaz F, Ersoz G, Zeytunlu M, Kilic M, Coker A. A rare cause of recurrent gastrointestinal bleeding: mesenteric hemangioma. World J Emerg Surg 2009;4:5.

9. Amati AL, Hecker A, Schwandner T, Ghanem H, Holler 
J, Reichert M, Padberg W. A hemangioma of the sigmoid colon mesentery presenting as a retroperitonealtumor: a case report and review. World J Surg Oncol 2014;12:79.

10. Parker WT, Harper JG, Rivera DE, Holsten SB, Bowden T. Mesenteric cavernous hemangioma involving small bowel and appendix: a rare presentation of a vascular tumor. Am Surg 2009;75:811-6.

11. Takamura M, Murakami T, Kurachi H, Kim T, Enomoto T, Narumi Y, Nakamura H. MR imaging of mesenteric hemangioma: a case report. Radiat Med 2000;18:67-9.

12. Yang GZ, Li J, Jin H. Giant mesenteric hemangioma of

Cite this article as: Esposito F, Di Serafino M, Severino R, Vafina Z, Ferrara D, Zeccolini M. Mesenteric infantile hemangioma: diagnostic work-up. Quant Imaging Med Surg 2018;8(2):252-256. doi: 10.21037/qims.2017.09.01 cavernous and venous mixed type: a rare case report. BMC Surg 2013;13:50.

13. Tan ST, Itinteang T, Leadbitter P. Low-dose propranolol for multiple hepatic and cutaneous hemangiomas with deranged liver function. Pediatrics 2011;127:e772-6.

14. Fette A. Propranolol in use for treatment of complex infant hemangiomas: literature review regarding current guidelines for preassessment and standards of care before initiation of therapy. ScientificWorldJournal 2013;2013:850193. 